\title{
Técnica de "socket-shield" para la preservación de reborde alveolar. Reporte de caso clínico.
}

\section{Socket-shield technique for the preservation of alveolar ridge. Clinical case report.}

\author{
Jorge Mija-Gómez ${ }^{1}$, Frank Paredes-Nomberto ${ }^{1}$, Yuri Castro-Rodríguez ${ }^{2}$
}

\author{
1. Especialidad de Periodoncia. Universidad \\ Nacional Mayor de San Marcos. Lima. Perú. \\ 2. Magister en Educación. Universidad Privada \\ Juan Pablo II. Lima. Perú. \\ * Correspondencia autor: Yuri Alejandro Castro \\ Rodríguez | Dirección: Jr. Tomás Catari 463, \\ Urb. El Trébol. Dpto. 201. Los Olivos. | E-mail: \\ yuricastro_16@hotmail.com | Celular991719062. \\ Trabajo recibido el 30/12/2017. \\ Aprobado para su publicación el 23/03/2019.
}

\begin{abstract}
RESUMEN
Algunas técnicas como la regeneración ósea guiada, uso de rellenos óseos y membranas se han planteado para limitar la reabsorción ósea post exodoncia. La técnica de "socket-shield" preserva un fragmento de raíz vestibular como medio para preservar la cortical ósea bucal. El presente reporte describe un caso clínico de fractura coronaria en un incisivo central superior que fue segmentado parcialmente y la porción vestibular de la raíz fue preservada para mantener la arquitectura de la cortical vestibular, al mismo tiempo se colocó un implante dental. Posterior a la rehabilitación fija, y con un seguimiento de un año se evidenció la preservación de los tejidos blandos periimplantarios a nivel de la mucosa y tejido óseo. No se observó alteraciones en la oseointegración y la pérdida ósea marginal periimplantaria fue nula. Se concluye que la técnica de "socket-shield" parece conservar clínicamente la arquitectura de la mucosa y el tejido óseo sin ocasionar patologías periimplantarias.
\end{abstract}

PALABRAS CLAVE

Implantes dentales; Alveolo dental; Regeneración ósea; Proceso alveolar..

Rev. Clin. Periodoncia Implantol. Rehabil. Oral Vol. 12(3); 154-156, 2019.

\section{ABSTRACT}

Some techniques such as guided bone regeneration with the use of bone fillings and membranes have been proposed to limit bone resorption. The socket-shield technique preserves a fragment of the vestibular root as a means of preserving the buccal bone cortex. This report describes a clinical case of coronary fracture in an upper central incisor that was partially segmented and the vestibular portion of the root was preserved to maintain the architecture of the vestibular cortex; at the same time, a dental implant with immediate provisionalization was placed. After the fixed rehabilitation and with a follow-up of one year, the preservation of the soft peri-implant tissues at the level of the mucosa and bone tissue was evidenced. No alterations in osseointegration were observed and marginal peri-implant bone loss was null. It is concluded that the socketshield technique seems to clinically conserve the architecture of the mucosa and bone tissue without causing peri-implant diseases.

\section{KEYWORDS}

Dental implants; Tooth socket; Bone regeneration; Alveolar process.

Rev. Clin. Periodoncia Implantol. Rehabil. Oral Vol. 12(3); 154-156, 2019.

\section{INTRODUCCIÓN}

Diversos biomateriales y métodos se han planteado para mantener o preservar el reborde alveolar luego de una extracción dentaria, especialmente la tabla vestibular ósea que suele reabsorberse y colapsar fácilmente. Se incluyen la colocación inmediata del implante dental luego de una exodoncia ${ }^{(1)}$, uso de rellenos óseos en el alveolo y el uso de membranas de barrera(2); sin embargo, estos procedimientos han demostrado mantener la dimensión del reborde alveolar en pocos casos y con bajas cantidades ${ }^{(3)}$. Aún no se ha reportado una completa preservación o una verdadera regeneración del alveolo dentario ${ }^{(4)}$

La reabsorción ósea se ha reportado en un promedio del $50 \%$ a nivel de ancho del reborde alveolar ${ }^{(5)}$ y de $2-4 \mathrm{~mm}^{(6)}$ en altura, siendo este proceso de reabsorción variable y no predecible. Un mayor grado de reabsorción se ha observado al elevar colgajos, biotipos gingivales delgados y raíces prominentes especialmente a nivel bucal(7). También se ha mencionado que la colocación inmediata de un implante dental no necesariamente previene la reabsorción del reborde alveolar ${ }^{(8)}$

La técnica de sumersión (inmersión) de raíz (TSR) fue descrita por Salama y cols. ${ }^{(9)}$, como una técnica que mantiene la raíz del diente en el alveolo para lograr preservar la mayor cantidad de tejido así como evitar la reabsorción de la cresta ósea, altura de la papila interdental y el ancho del reborde alveolar, además de mantener el aparato de inserción del diente.

Bajo este principio, mantener el fragmento bucal de la raíz del diente dentro del alveolo luego de una sección radicular de mesial a distal y extraer el resto del tejido radicular para la inserción de un implante dental permitiría preservar los tejidos como la técnica original y colocar un implante dental al mismo tiempo. Hürzeler y cols. ${ }^{(4)}$ demostraron que la conservación intencional de la porción bucal de la raíz puede ayudar a garantizar la preservación fisiológica de las estructuras óseas vestibulares y bucales si el implante se coloca en contacto con este fragmento natural de raíz (escudo). En el presente reporte 
se describe un caso clínico con diagnóstico de fractura coronaria a nivel de un incisivo central superior el cual fue extraído parcialmente dejando el fragmento radicular vestibular como "escudo" para preservar la arquitectura de la cortical vestibular, y la colocación inmediata de un implante dental.

\section{Informe del caso}

Paciente de 38 años de edad de sexo masculino, que acudió a la clínica de Periodoncia del Posgrado de la Facultad de Odontología de la Universidad Nacional Mayor de San Marcos (Lima. Perú) en junio del 2016 por presentar problemas estéticos durante la sonrisa, esto debido a la fractura de un diente en el sector antero superior. En la anamnesis, el paciente refiere no fumar ni presentar antecedentes sistémicos.

Al examen clínico intraoral se evidenció escasas lesiones cariosas y una leve inflamación gingival. A nivel del sector anterosuperior se observó al incisivo central superior derecho fracturado y con presencia de crecimiento gingival a nivel del fragmento radicular evidenciable al momento de sonreír (Figura $1 \mathrm{~A}$ y $1 \mathrm{~B}$ ). El examen radiográfico del sector anterior evidenció la ausencia de la corona clínica sin patologías a nivel de la zona periradicular (Figura 1C), el examen tomográfico mostró anchos y altura apropiadas para la colocación de un implante dental (Figura 1D).

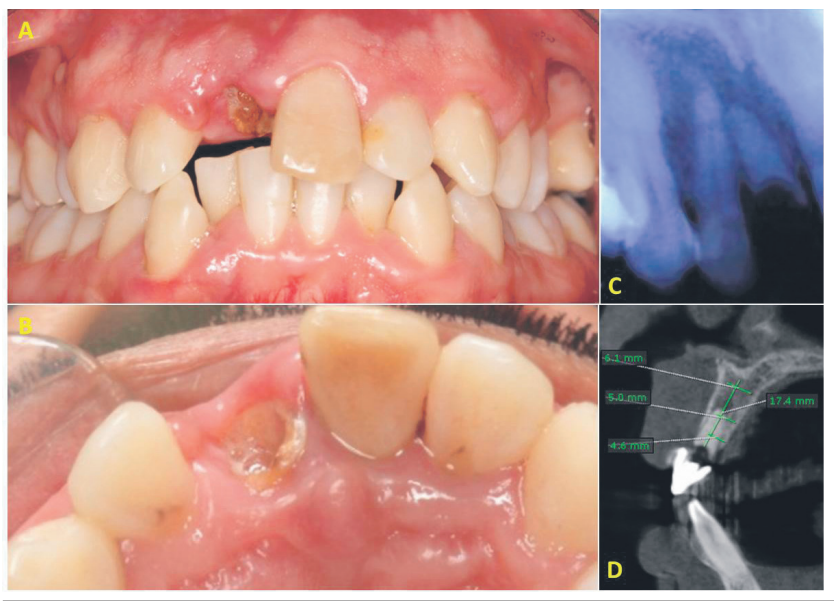

Figura 1. A: Vista frontal de la fractura coronaria en el incisivo central. B: Vista oclusal de la fractura y crecimiento gingival. C. Vista radiográfica del fragmento radicular. D: Vista tomográfica con adecuados ancho y altura para la colocación de implante dental.

El caso fue diagnosticado con una fractura corona-radicular del incisivo central superior, enfermedad gingival asociada a biofilm dental y exceso gingival a nivel del diente fracturado. Adicionalmente múltiples recesiones gingivales Miller tipo 1 fueron encontradas en caninos y premolares inferiores. Debido a la demanda estética del caso y de la exigencia del paciente se planificó la exodoncia parcial del diente fracturado a través de la técnica de "socket-shield" y la colocación inmediata de un implante dental.

El paciente fue notificado con las opciones de tratamiento y luego de firmado el consentimiento informado se procedió a realizar los siguientes tratamientos: FASE 1: Educación sobre el biofilm así como motivación en la técnica de cepillado, control de biofilm dental, instrucciones de higiene oral, raspado y alisado radicular, eliminación de elementos retenedores de placa bacteriana. FASE 2: Exodoncia parcial del incisivo central superior y colocación de implante dental inmediato. FASE 3: Confección de pilar personalizado, diseño de sonrisa digital para dar mejor proporción de piezas a rehabilitar y coronas metal cerámica sobre el implante y el incisivo central izquierdo.

\section{Procedimiento quirúrgico}

La zona a operar se infiltró con anestesia local, lidocaína $2 \%$ más epinefrina 1:80000 (Scandicaine ${ }^{\circledR}$, Septodont. Japon). Se diseñó una incisión sulcular a nivel palatino del incisivo central superior seguida de la reducción de la porción coronaria de la raíz (Figura 2A), luego se procedió a realizar el protocolo de fresado para la colocación de un implante dental; se inició con la fresa lanza y luego la fresa piloto. El protocolo de fresado (Kit quirúrgico Tapered ${ }^{\circledR}$ Biohorizons. U.S.A) fue iniciado para obtener la orientación de colocación del implante dental y la cantidad de fragmento radicular a extraer (Figura 2B y 2C). Luego de las dos primeras fresas se procedió a hemisectar la raíz utilizando una fresa de fisura en dirección mesio-distal, se realizó una extracción atraumática del segmento palatino de la raíz (Figura 2D). El segmento bucal remanente fue reducido utilizando una fresa quirúrgica dejando una delgada capa de la raíz manteniendo intacta la cortical ósea vestibular (Figura 2E). Luego de extraído el fragmento radicular se procedió a realizar un tercer fresado según el protocolo para luego instalar un implante dental (Tapered Internal $\AA^{\circledR}$, Biohorizons. U.S.A) con unas longitudes de 3,8 mm x $12 \mathrm{~mm}$ en el alveolo dejando $1 \mathrm{~mm}$ de espacio entre la superficie vestibular del implante y el fragmento radicular (Figura 2F). Se dejó con la tapa de cicatrización de 3 $\mathrm{mm}$ de cuff. Se colocó fibrina rica en plaquetas según el protocolo Choukroun y cols. ${ }^{(10)}$ entre el implante dental y el fragmento radicular (Figura 2G) para luego suturar con ácido pologlicólico (Sinorgmed $₫$, Alemania) y puntos cruzados (Figura $2 \mathrm{H}$ ). Se recomendó una dieta blanda luego de la cirugía de colocación de implante dental más antiinflamatorios (diclofenaco $50 \mathrm{mg}$ cada 8 horas por cinco días) y antibioticoterapia (azitromicina en tabletas de $500 \mathrm{mg}$ cada 24 horas por tres días; se optó por este régimen debido a su elevada concentración del fármaco en los tejidos óseos alveolares $\left.{ }^{(11)}\right)$. No se utilizó provisionalización inmediata para no interferir con el proceso de cicatrización a nivel de la zona vestibular del implante dental colocado. La terapia antibiótica fue prescrita para reducir la carga bacteriana posoperatoria en la zona intervenida.

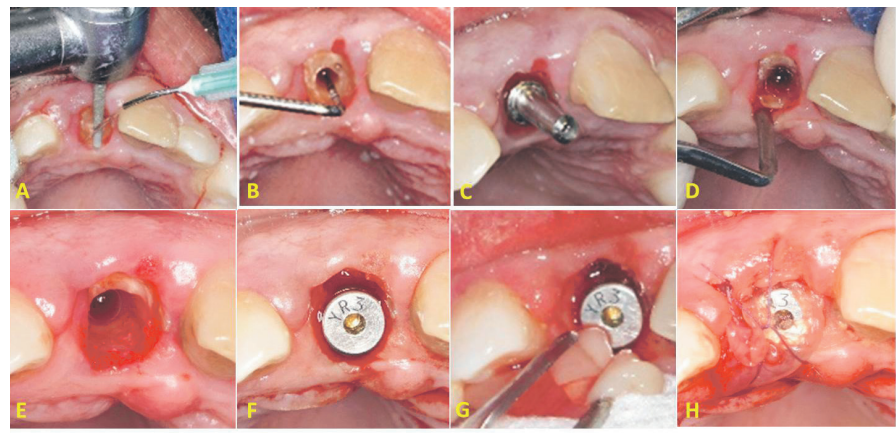

Figura 2. A: Reducción de la porción coronaria de la raíz. B: Primera y segunda perforación con fresa lanza y piloto. C: Verificación del fresado con pin de paralelismo. D: Luxación del fragmento palatino y exodoncia parcial. E: Reducción del fragmento radicular vestibular con fresa redonda. F: Colocación de implante dental a $1 \mathrm{~mm}$ del fragmento radicular vestibular. G: Colocación e fibrina rica en plaquetas en el espacio entre implante y fragmento radicular. $\mathrm{H}$ : Sutura en punto cruzado.

\section{Seguimiento clínico}

Luego de 10 días de posoperatorio la cicatrización del tejido blando fue adecuada no observando complicaciones (Figura 3A). Luego de seis meses de cicatrización no se observó movilidad del implante dental (Figura 3B), tampoco de imágenes radiolúcidas alrededor del implante dental (Figuras $3 \mathrm{C}$ y $3 \mathrm{D}$ ); por el contrario se evidenció que el grosor de la cortical vestibular se conservo en $1 \mathrm{~mm}$ (Figura 3D). Se procedió a instalar un pilar y corona provisional (Figura $3 E$ y $3 F$ ), en la cual con incrementos de resina en la zona crítica y subcrítica se optimizó el perfil de emergencia (Figura 3G). Colocado el pilar definitivo en el implante se procedió a cementar una corona metal cerámica (Figuras $3 \mathrm{H}$ y $3 \mathrm{I}$ ). Se optó por la cementación debido a que son pocas las diferencias entre coronas cementadas o atornilladas sobre el comportamiento del hueso marginal peri-implante y del tejido blando periimplantario en el sector anterior. Luego de seis meses de control se registró un segundo examen tomográfico. Se evidenció la preservación del ancho del reborde alveolar, así como la altura y grosor de la cortical vestibular sin patologías a nivel del fragmento radicular vestibular. Las mediciones tomográficas fueron realizadas desde el borde más vestibular del hueso maxilar hasta la superficie más externa del implante en los puntos cervical, medio y apical (Figura 3D).

\section{DISCUSIÓN}

Además de afectar el resultado estético, la atrofia del reborde alveolar después de la extracción dental tiene, sobre todo, un impacto negativo en la posterior restauración protésica.

Idealmente, la prevención de la reabsorción de cresta alveolar debería ser un método económico y mínimamente invasivo, con requisitos mínimos de material. Sin embargo, estos criterios no se cumplen completamente con ninguno de los métodos disponibles en la actualidad. Además, el objetivo de la preservación de la cresta alveolar después de la extracción dental ni siquiera se ha alcanzado con técnicas sofisticadas. Por lo tanto, la búsqueda de conceptos simples con resultados predecibles todavía está en la investigación actual.

Con la técnica de sumersión de la raíz (TSR), la retención del ligamento periodontal a través del fragmento radicular ("shield") virtualmente podría eliminar la reabsorción ósea ${ }^{(9)}$. Además, la estabilización apropiada del coágulo entre el implante y el escudo facilitaría la neoformación ósea a largo plazo. Las alternativas implantológicas en esta técnica incluyen colocar el implante dental luego del proceso de cicatrización, colocar inmediatamente a la preparación del escudo o la colocación de pónticos en el reborde alveolar. En el presente caso se optó por la técnica de "socket-shield" como una alternativa para evitar 


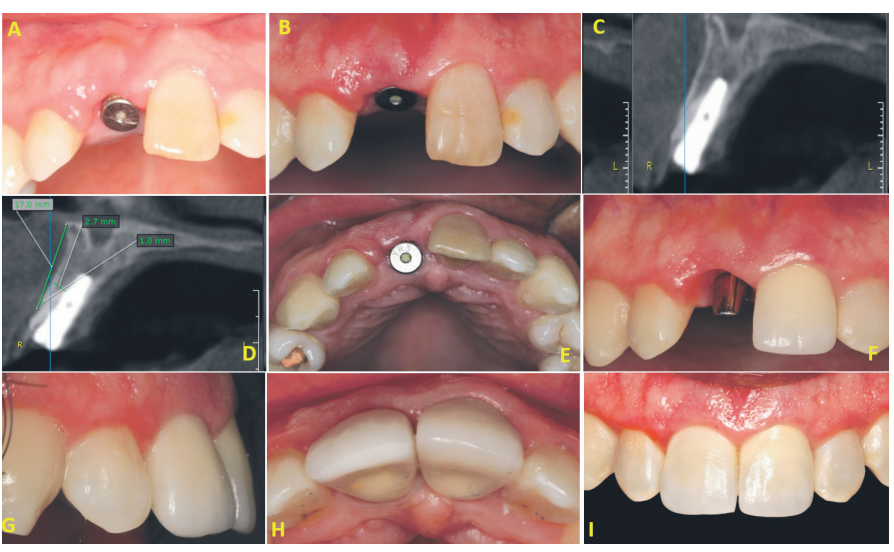

Figura 3. A: Control posoperatorio a los diez días. B: Vista frontal de la apariencia clínica vestibular. C: Vista tomográfica sin anomalías en el fragmento radicular. D: Mediciones de la cortical vestibular preservada. E: Vista oclusal con el cicatrizal colocado. F: Colocación del pilar definitivo. G: Vista lateral de las coronas metal cerámica. H: Vista oclusal de la corona y reborde alveolar. I: Vista frontal de las coronas cementadas.

el colapso de la cortical vestibular luego de la exodoncia dentaria y así no alterar el resultado estético de la rehabilitación definitiva; estudios avalan que esta técnica ha demostrado alto potencial en la prevención de la reabsorción del tejido bucal en reportes clínicos ${ }^{(10)}$.

Algunos estudios han evaluado la seguridad de las raíces sumergidas en el hueso alveolar y concluyen que estas pueden mantenerse siempre que no ocurran infecciones o se movilicen debido a que podrían actuar como cuerpos extraños y ser un nido de infección o migración; sin embargo, podrían ser un elemento que preserve el tejido óseo y las dimensiones del tejido blando. Filippi y cols. ${ }^{(12)}$ mencionan que la decoronación e inmersión radicular de dientes anquilosados es una técnica quirúrgica simple y segura que permite preservar el hueso alveolar antes de la colocación de un implante dental. Salama y cols. ${ }^{(9)}$ también acotan que la TSR no solo elimina el riesgo de caries y periodontitis, sino que la retención de una raíz permite preservar al máximo el hueso alveolar circundante y el tejido blando. La retención del escudo bucal de la raíz durante la colocación del implante no parece interferir con la osteointegración y puede ser beneficiosa para preservar la cortical ósea bucal (4)

Clínicamente se ha observado que ofrece una estabilidad clínica de los contornos de los tejidos blandos durante los 3 años de seguimiento ${ }^{(13)}$. Por tal motivo puede ofrecer una opción de tratamiento factible para los dientes con fracturas verticales ${ }^{(14)}$.

La técnica representa un enfoque alternativo para intervenir procesos de remodelación y reabsorción por el mantenimiento de la parte facial de la raíz durante la extracción dental. La colocación inmediata de un implante soporta el fragmento de la raíz facial y, por lo tanto, impide el colapso de la pared bucal(15).
Dentro de sus características se incluye el ofrecer una menor invasividad en el momento de la cirugía y resultados estéticos con una preservación eficaz de los contornos del tejido facial(16). Con un seguimiento clínico de un año, el análisis tomográfico de nuestro caso evidenció el mantenimiento del grosor del reborde alveolar, la no modificación del grosor y altura de la cortical vestibular y la ausencia de imágenes patológicas. Esto concuerda con los estudios pre clínicos que han demostrado que el fragmento radicular bucal no interfiere con el proceso de oseointegración del implante dental y que es beneficioso en la preservación de la cortical bucal ósea(4).

Los resultados clínicos muestran que la técnica de "socket-shield" cumple esencialmente las expectativas de un método ideal para prevenir la reabsorción de la cresta alveolar y los requisitos mínimos de un biomaterial. Algunos autores mencionan incluso que el cemento del fragmento radicular puede integrarse al implante dental ${ }^{(17)}$. También se ha reportado que el coágulo sanguíneo entre el implante y el fragmento radicular actúa como barrera que evita la migración epitelial y favorece la regeneración ósea(4). En nuestro caso se optó por utilizar fibrina rica en plaquetas (PRF) como biomaterial del tipo agente bioactivo para evitar la migración apical del epitelio así como favorecer la cicatrización del tejido blando periimplantario, dado que es bien conocido que los concentrados plaquetarios pueden promover la quimiotaxis, migración celular, angiogénesis, proliferación, diferenciación y producción de matriz extracelular ${ }^{(18)}$, esto puede conllevar a una aceleración de la formación de hueso, remodelado óseo y cicatrización de heridas ${ }^{(19)}$

Es necesario destacar que la extracción parcial reportada es sensible a la técnica y puede estar asociada con el riesgo de desplazamiento del fragmento radicular bucal, o incluso de la cortical vestibular. Luego del momento de la extracción, el fragmento de raíz debe reducirse a lo largo de su eje vertical hasta el nivel de la altura de la cresta alveolar para evitar la perforación de la mucosa bucal durante el período de cicatrización. En caso de que el fragmento de raíz se exponga, a pesar de esta medida preventiva, la reducción de altura y la renovación del tejido se pueden realizar durante el control postoperatorio.

\section{CONCLUSIÓN}

En base a lo observado del caso podemos concluir que mantener la superficie bucal de la raíz en unión a la colocación inmediata de un implante dental fue una técnica que logró la oseointegración del implante colocado sin una respuesta inflamatoria observable. En contraste a otras técnicas más complejas e invasivas, la técnica de "socket-shield" del presente caso resultó ser más económica y nos permitió evitar la reabsorción de la cortical vestibular.

\section{CONFLICTO DE INTERESES}

Los autores declaran no tener conflictos de interés.

\section{FUENTE DE FINANCIAMIENTO}

Autofinanciado.

\section{BIBLIOGRAFÍA}

1. Botticelli D, Berglundh T, Lindhe J. Hard-tissue alterations following immediate implant placement in extraction sites. J Clin Periodontol. 2004;31:820-8.

2. Lekovic V, Carmargo P, Klokkevold P, Weinlaender M, Kenney E, Dimitrijevic B, et al. Preservation of alveolar bone in extraction sockets using bioabsorbable membranes. $J$ Periodontol. 1998;69:1044-9.

3. Araújo M, Linder E, Lindhe J. Effect of a xenograft on early bone formation in extraction sockets: an experimental study in dog. Clin Oral Implants Res. 2009;20:1-6.

4. Hürzeler MB, Zuhr O, Schupbach P, Rebele SF, Emmanouilidis N, Fickl S. The socket-shield technique: a proof-of-principle report. J Clin Periodontol. 2010;37:855-62. 5. Schropp L, Wenzel A, Kostopoulos L. Bone healing and soft tissue contour changes following single-tooth extraction: A clinical and radiographic 12-month prospective study. Int J Periodontics Restor Dent. 2003;23:313-23.

6. Araújo MG, Lindhe J. Dimensional ridge alterations following tooth extraction: An experimental study in the dog. J Periodontol. 2005;32:212-8.

7. Hämmerle CH, Araújo MG, Simion M. Osteology consens group 2011. Evidencebased knowledge on the biology and treatment of extraction sockets. Clin Oral Implant Res. 2012;23:80-2.

8. Araújo MG, Sukekava F, Wennström JL, Lindhe J. Ridge alterations following implant placement in fresh extraction sockets: An experimental study in the dog. J Periodontol. 2005;32:645-52.

9. Salama T, Ishikawa H, Salama A, Funato D, Garber A. Advantages of the root submergence technique for pontic site development in esthetic implant therapy. Int $\mathrm{J}$ Periodontics Restor Dent. 2007;27(6):521-7.

10. Glocker M, Attin T, Schmidlin P. Ridge Preservation with Modified "Socket-shield" Technique: a methodological case series. Dent J. 2014;2:11-21.

11. Escalante MG, Eubank TD, Leblebicioglu B, Walters J. Comparison of azithromycin and amoxicillin prior to dental implant placement: an exploratory study of bioavailability and resolution of postoperative inflammation. J Periodontol. 2015;86(11):1190-200.
12. Filippi A, Pohl Y, von Arx T. Decoronation of an ankylosed tooth for preservation of alveolar bone prior to implant placement. Dent Traumatol. 2001;17:93-5.

13. Mitsias ME, Siormpas KD, Kontsiotou-Siormpa E, Prasad H, Garber D, Kotsakis GA. A step-by-step description of PDL-Mediated ridge preservation for immediate implant rehabilitation in the esthetic region. Int $\mathrm{J}$ Periodontics Restorative Dent. 2015;35(6):835-41.

14. Bäumer $D$, Zuhr $O$, Rebele $S$, Schneider $D$, Schupbach $P$, Hürzeler $M$. The socket-shield technique: first histological, clinical, and volumetrical observations after separation of the buccal tooth segment - a pilot study. Clin Implant Dent Relat Res. 2015:17(1):71-82.

15. Saeidi Pour R, Zuhr O, Hürzeler M, Prandtner O, Rafael CF, Edelhoff D, et al. Clinical benefits of the immediate implant socket shield technique. J Esthet Restor Dent. 2017;29(2):93-101.

16. Bäumer D, Zuhr O, Rebele S, Hürzeler M. Socket shield technique for immediate implant placement - clinical, radiographic and volumetric data after 5 years. Clin Oral Implants Res. 2017;28(11):1450-8

17. Buser D, Warrer K, Karring T. Formation of a periodontal ligament around titanium implants. J Periodontol. 1990;61:597-601.

18. Tanaka H, Toyoshima T, Atsuta I, Ayukawa Y, Sasaki M, Matsushita Y, et al. Additional effects of platelet-rich fibrin on bone regeneration in sinus augmentation with deproteinized bovine bone mineral: preliminary results. Implant Dent 2015;24(6):669-74

19. Ali S, Bakry SA, Abd-Elhakam H. Platelet-rich fibrin in maxillary sinus augmentation: a systematic review. J Oral Implantol. 2015;41(6):746-53. 